\title{
COMMISSION 7 : CELESTIAL MECHANICS (MÉCANIQUE CELESTE)
}

Report of Meetings, 24, 27 and 29 July 1991

PRESIDENT : J. Henrard

VICE-PRESIDENT : A. Deprit

SECRETARY : S. Ferraz-Mello

\section{Business Meeting}

The business session was held on July 24th. A second brief meeting was held on July 29th. Items addressed were:

\section{Election of Organizing Committee}

The commission elected the following officers and members of the Organizing Committee for the term 1988 to 1991 :

$\begin{array}{ll}\text { President: } & \text { A.Deprit } \\ \text { Vice President: } & \text { S.Ferraz-Mello } \\ \text { Members: } & \text { K.B.Bhatnagar } \\ & \text { J.Chapront } \\ & \text { Cl.Froeschlé } \\ & \text { J.Henrard (Past President) } \\ & \text { K.Kholshevnikov } \\ & \text { H.Kinoshita } \\ \text { J.H.Lieske } & \text { He Miaofu } \\ & \text { A.Milani } \\ \text { S.Peale } & \text { A.E.Roy } \\ \text { A.G.Sokolsky }\end{array}$

\section{Election of New Members of the Commission}

The following IAU members were proposed as new members of Commission 7 and were approved after a short presentation: R.Branham (Argentina), C.Edelman (France), L. El Bakali (Morocco), Z.Knežević (Yugoslavia), Xu Jihong (China), R.Orellana (Argentina), M.E.Soffel (Germany), A.G.Sokolsky (URSS), G.B.Valsecchi (Italy), R. Vieira Martins (Brazil) and Liao Xinhao (China). The proposals were done by the National Committees or by members of the Commission present at the meetings. 


\section{Consultants}

The Commission elected the following consultants for the term 1988-1991: V.I.Arnold (USSR), A.Chenciner (France), K.Meyer (USA), J.Moser (Switzerland), D.Saari (USA) and C.Simó (Spain).

\section{Deceased Members}

The commission paid tribute to the memory of the distinguished scientists devoted to Celestial Mechanics Drs. Leland E. Cunningham, Edgard Everhart and John P. Vinti, members of the commission, and John V. Breakwell, deceased since the past General Assembly.

\section{Working Group on Near Earth Objects}

In the first session, Dr. B.Marsden reported to the commission the contents of the proposals concerning the support to programs designated to study near-earth objects. In the following session the Commission discussed and approved the creation of an ad-hoc Working Group on natural near-Earth objects (NEO) and indicated Drs. P.K.Seidelman and A.G.Sokolsky to represent the commission in this Working Group.

\section{Recommendations on Reference Systems}

The commission participated in the joint discussion held on July 25th.

\section{Past and future Colloquia and Symposia}

Reports were presented concerning IAU Colloquium No. 132 held in Delhi (India) in October, 1990 and IAU Symposium No. 152 held in Angra dos Reis (Brazil), in July 1991. The president of the Commission informed that IAU Colloquium No. 118, scheduled to be realized in June 1989, in China, has been postponed because of the internal political situation of China at that time and then cancelled because of the low number of people that confirmed their participation in the meeting at the new scheduled date (mid 1990). Two possible proposals of Colloquia for 1994, one in URSS and the other in Belgium were announced. Informations were also given on meetings interesting this commission to be held in 1992 in France and in Czechoslovakia.

\section{Scientific Sessions}

Five invited reports were presented in the sessions held on July 27 th and 29 th:

A. Present status of rigid Earth rotation theory (H.Kinoshita)

B. The stellar problem of three bodies (S.Ferrer)

C. Chaotic motion in the Solar system. A review. (J.Laskar)

D. Integration of elliptic functions by computer (A.Deprit)

E. The scientific projects of ITA on the computer methods of Celestial Mechanics (A.G.Sokolsky) 
The following abstracts were provided by the lecturers.

\section{Present Status of Rigid Earth Rotation Theory (H.Kinoshita)}

The present IAU Nutation Series, which is based on Kinoshita's (1976) rigid Earth theory modified by Wahr's (1979) linear response theory for the non-rigidity of the Earth, is now not compatible with the recent precise observations such as VLBI and LLR. In these circumstances, Kinoshita and Souchay (1990) have reconstructed a rigid Earth rotation theory by taking account of

1) more precise orbital theories of the Moon and the Sun,

2) both direct and indirect planetary perturbations and

3) the coupling effect between the rotational motion and the orbital motion.

The truncation level of the new trigonometric series is 0.005 millisecond of arc.

Then Souchay and Kinoshita (1991) compared the new nutation series with numerical integration and showed that the internal accuracy of the new rigid rotation theory is 0.1 millisecond of arc, which is much better than the precision of the present observations. They found, however, that the residuals in the comparison still have systematic components which correspond to the term with argument $\Omega$, the semi-monthly term, and the semi- annual term.

\section{The Stellar Problem of Three Bodies (S.Ferrer)}

Presentation of a research did jointly with Dr. Osácar to clarify and sharpen the qualitative analysis of this problem made by Lidov and Ziglin. The authors explained how an extensive symbolic reduction carried by computer led them to realize that the reduced phase space is a two-dimensional sphere. The diagrams corresponding to each type of phase flow according to a complete discussion of all possible bifurcations determined by the four integrals of the system were presented.

\section{Chaotic Motion in the Solar System: a Review (J.Laskar)}

The number of publications on chaotic behaviour in the solar system has increased very much in the previous years. It reflects that we begin now to really take into account the result of Poincare that the equations of celestial mechanics are not integrable and this consideration necessitate for us the use of more sophisticated mathematics. One of the first evidence of chaotic motion in the solar system was the chaotic tumbling of Hyperion. In case of much smaller perturbations, it is possible to trap the chaotic region between KAM invariant curves, in order to obtain stability for all time. Even in a chaotic region, like the vicinity of an unstable Lagrangian point, the computation of invariant manifolds allows to make precise orbit determination and satellites station keeping. In the vicinity of a stable Lagrangian point, improved Nekhoroshev's theorems give regions of practical stability of significant size. Secondary resonances are found to play an important role in the tidal evolution of the Uranian satellites in order to allow Miranda to increase its inclination and then escape from resonance. The solar system and in particular the inner solar system was found to be chaotic because of secular resonances, with a Lyapunov exponent of $1 / 5$ million years. This has been confirmed by direct numerical integration. Ref: Chaotic Behaviour of the solar system (J. Laskar) Transactions Vol. 21 A (Commission 7 report). 


\section{Integration of Elliptic Functions by Computer (A.Deprit)}

In collaboration with Prof. Alberto Abad, Prof. Vincent Coppola and Dr. Bruce Miller, a code in Mathematica to calculate in symbolic form the integral of a product of powers, positive or negative, of Jacobian elliptic functions has been developed. The author pointed to some of the problems in celestial mechanics where an automated processor of elliptic functions and elliptic integrals is likely to open new vistas.

\section{The Scientific Projects of I.T.A. on the Computer Methods of Celestial Mechanics (A.G.Sokolsky)}

Some important problems in Celestial Mechanics are described: algorithmization and revision of local methods; quasiglobal methods; global (evolutionary) models; economization of algorithms and the theory of potential; connection with ephemeris astronomy. All such problems lead us to the idea that we need new approaches in computer realization of modern methods of astronomy. The main tools of this informational technology are:

1. In the field of computational algorithms the basic efforts must be concentrated on the construction of new fast integrators which can be adapted to the concrete form of integrated equations; the construction of summators for long analytical expressions; the application of modern observational data processing and filters well suited for astronomy; the accomplishment of parallel computations.

2. In the field of analytic calculations, there are two important problems - how to find the equilibrium point between universal CAS (computer algebra systems) and specialized CAS which are constructed for specific narrow classes of tasks; how to organize an interface between numeric and analytical components of algorithms.

3. In the field of representation of voluminous arrays of observational data in the computer form, one should think in terms of data bases. Modern astronomical data bases must include: banks of observational data, banks of theories, banks of requests.

4. At last, the modern program product must have a developed user interface which includes the shell (help-files, the menu subsystem, etc.) and gives the opportunity for the user to educate himself when using this program product.

As examples of realization of such an approach some computer products of ITA are described:

"STAMP" - electronic book for representation of ephemerides of minor planets for the current year. This program product is a computer version of the known year-book "Ephemerides of Minor Planets", published by ITA in the last 45 years.

"CERES" - integrated program system for studies of minor planets of the Solar system. It allows to work with data bases of numbered minor planets, to accomplish ephemeris computations, attachment to the observations, loaning from the fundamental stellar catalog, visualization of results, use of an informational help-subsystem.

"CELESTA" - integrated package for application of local methods of nonlinear analysis to the problems of classical and celestial mechanics. It contains special procedures of the REDUCE CAS and a shell implementing the user-interface. 